\title{
Assessment of disintegrant efficacy with fractal dimensions from real-time MRI
}

\author{
Julian Quodbach ${ }^{\mathrm{a}}$, Amir Moussavi ${ }^{\mathrm{b}}$, Roland Tammer ${ }^{\mathrm{b}, \mathrm{c}}$, Jens Frahm ${ }^{\mathrm{b}, \mathrm{c}}$, \\ Peter Kleinebudde ${ }^{\text {a,* }}$ \\ a Institute of Pharmaceutics and Biopharmaceutics, Heinrich-Heine-University, Universitaetsstasse 1, Duesseldorf 40225, Germany \\ ${ }^{\mathrm{b}}$ Biomedizinische NMR Forschungs GmbH am Max-Planck-Institut für Biophysikalische Chemie, Am Fassberg 11, Goettingen 37070, Germany \\ ${ }^{\mathrm{c}}$ Center for Nanoscale Microscopy and Molecular Physiology of the Brain (CNMPB), Goettingen 37070, Germany
}

\section{A R T I C L E I N F O}

Article history:

Received 28 July 2014

Received in revised form 9 September 2014

Accepted 11 September 2014

Available online 16 September 2014

Keywords:

Formulation

Polymers

Visualization

Superdisintegrants

Tablet disintegration

\begin{abstract}
A B S T R A C T
An efficient disintegrant is capable of breaking up a tablet in the smallest possible particles in the shortest time. Until now, comparative data on the efficacy of different disintegrants is based on dissolution studies or the disintegration time. Extending these approaches, this study introduces a method, which defines the evolution of fractal dimensions of tablets as surrogate parameter for the available surface area. Fractal dimensions are a measure for the tortuosity of a line, in this case the upper surface of a disintegrating tablet. High-resolution real-time MRI was used to record videos of disintegrating tablets. The acquired video images were processed to depict the upper surface of the tablets and a box-counting algorithm was used to estimate the fractal dimensions. The influence of six different disintegrants, of different relative tablet density, and increasing disintegrant concentration was investigated to evaluate the performance of the novel method. Changing relative densities hardly affect the progression of fractal dimensions, whereas an increase in disintegrant concentration causes increasing fractal dimensions during disintegration, which are also reached quicker. Different disintegrants display only minor differences in the maximal fractal dimension, yet the kinetic in which the maximum is reached allows a differentiation and classification of disintegrants.
\end{abstract}

(c) 2014 Elsevier B.V. All rights reserved.

\section{Introduction}

Disintegrants in a pharmaceutical formulation promote the disruption of the tablet into smaller particles. The aim is to generate as small as possible particles in the least amount of time. This process increases the wettable surface area and thereby accelerates the release of the incorporated drug substance. In general, modified starch, cellulose, and synthetic polymers are used as disintegrants. The polymer chains of the natural polymers are cross-linked to achieve insolubility in the disintegration medium and carboxylated to increase the hydrophilic properties. Polacrilin potassium, a cross-linked co-polymer of methacrylic acid and divinyl benzene, and crospovidone, cross-linked polyvinylpyrrolidone, are commonly used synthetic polymers. They are included in many marketed products and part of research projects (Jyothi and Lakshmi, 2011; Soumya, 2013).

\footnotetext{
* Corresponding author. Tel.: +49 21181 14220; fax: +49 2118114251

E-mail addresses: kleinebudde@hhu.de, kleinebudde@uni-duesseldorf.de (P. Kleinebudde)
}

Even though the choice of the disintegrant can significantly influence the drug dissolution rate, no comparative data is available on the efficacy in generating the smallest possible particles. The dissolution rate is linked to the available surface area of a drug and is frequently used to find the optimal disintegrant for a formulation. Nevertheless, several studies investigated other parameters such as the disintegration time to quantify the effectiveness of disintegrants.

Commonly, the disintegration test according to the European Pharmacopeia is used, which measures the time point at which all remainders of a tablet completely pass through a moving sieve with a $2 \mathrm{~mm}$ aperture submersed in water. Gissinger and Stamm compared water absorption, contact angle, swelling volume, and disintegration times of a range of disintegrants (Gissinger and Stamm, 1980). They ranked the efficacy mainly according to the disintegration time and concluded that modified starch and cellulose, low-substituted hydroxypropylcellulose, polacrilin potassium, and crospovidone are superior to other disintegrants. The impact of the aforementioned factors was only briefly discussed and no preferable values were mentioned. Rudnic et al. compared disintegration times of tablets with a dibasic 
calcium phosphate and lactose matrix (Rudnic et al., 1982). Again, modified starch and modified cellulose, polacrilin potassium, and crospovidone as well as an anionic cellulose polymer proved to be more efficient in terms of disintegration time than unmodified natural cellulose. Even though the disintegration time is used as a measure of disintegrant efficacy, it primarily represents a qualitative attribute which offers only limited information about the true effectiveness of disintegrants.

Dissolution studies are also used to quantify a disintegration process. Although these tests yield qualitative and quantitative data of the released drug amount, they are subject to influences by a large number of factors. Gordon and Chowhan, for example, examined the influence of storage conditions on the dissolution rate of 4-aminobenzoic acid tablets containing different disintegrants and matrix substances of varying solubility and hygroscopicity (Gordon and Chowhan, 1990). The variations of the dissolution rates were mainly influenced by the matrix substances and less pronounced between different disintegrants. Croscarmellose sodium and crospovidone yielded the highest dissolution rates after $5 \mathrm{~min}$ in all matrix substances. In the case of a sorbitol matrix, however, the tablets containing sodium starch glycolate released less API than the tablet without disintegrant. The dissolution rate was also used as parameter for disintegrant efficacy by Chen et al. (Chen et al., 1997), who investigated the impact of the disintegration medium on the behavior of acetaminophen tablets with crospovidone, sodium starch glycolate, and croscarmellose sodium. Crospovidone proved to be more robust against a $\mathrm{pH}$ change of the disintegration medium compared to croscarmellose sodium and sodium starch glycolate. The dissolution rate was highest for sodium starch glycolate and sodium starch glycolate and lowest for crospovidone.

The studies demonstrate that dissolution data reflect many different factors and not only disintegrant characteristics, which renders dissolution insufficient for disintegrant characterization. Also, a detectable substance is always required for dissolution quantification. Therefore, these studies do not depict the action of the disintegrant alone and also do not consider the available surface area of the resulting particles. Interactions between excipients and drug substance might also cause deviations in the release profiles that are not connected with the used disintegrant. Additionally, dissolution studies are not capable of detecting rapid disintegration processes presented by modern dosage forms (e.g., oral disintegrating tablets).

Another approach was developed by Caramella (Caramella, 1990), who simultaneously measured the water uptake and force development of disintegrating tablets. Caramella then defined the "derivative of the force-water curve at the center of the distribution" as the "maximum capability of the compact to transform water into force" and named the parameter "force equivalent" when a sigmoidal curve was observed. Iwao et al. introduced a novel method to derive the available surface area from dissolution studies (Iwao et al., 2013). They calculated the available surface area during dissolution based on the dissolution rate of the drug from a specific area. The highest available surface areas were found for crospovidone and croscarmellose sodium in concentrations of $5 \%$.

Moreover, a direct measurement of the resulting particle sizes is complicated due to the fragile structures of the wetted particles. Shotton and Leonard utilized a custom built wet sieving apparatus to evaluate the resulting particles of a disintegration process (Shotton and Leonard, 1972). They investigated the influence of the mode of disintegrant incorporation in tablets made from wet-massed granules with maize starch as disintegrant. As they did not sufficiently describe the wet sieving procedure, results of this study should only be interpreted with care. Zhao and

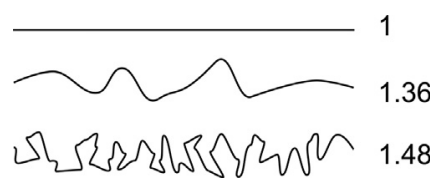

Fig. 1. Concept of fractal dimensions.

Augsburger reported unsuccessful attempts to determine the size of particles created during the disintegration process with optical microscopy, laser scattering, and sieving (Zhao and Augsburger, 2005). Mesnier et al. introduced a flow cell in which a disintegrating tablet is filmed with a digital camera (Mesnier et al., 2013). The video was analyzed in terms of tablet thickness, diameter, and size and number of detached particles. However, the setup is limited to the analysis of slowly eroding tablets and not for the evaluation of rapidly disintegrating tablets.

In this study, fractal dimensions (FDs) are proposed as a novel surrogate parameter for the accessible surface area of tablets and as a means to compare disintegrant efficacy. Mandelbrot introduced FDs to describe irregularly shaped coastlines (Mandelbrot, 1967) and pointed out that "geographical curves are so involved in their detail that their lengths are often infinite or, rather, undefinable." The smaller the chosen measurement scale, the longer is the length of an object boundary, leading to infinite length at an infinitely small measurement scale. The concept of Euclidean space cannot sufficiently describe these curves. Therefore, FDs are calculated and ascribed to crooked curves and surfaces. A straight line still has a dimension of $D=1$, but an irregular curve presents with a dimension between 1 and 2 . The more irregularly shaped a curve, the closer the FD is to 2 (Fig. 1). In consequence, curves with higher FDs possess a greater apparent length at a given resolution. FDs are constant values, which describe geometric characteristics of objects. This means that the absolute length of a fractal line depends on the number of generations over which fractal characteristics are present. The boundary length increases when the measurement scale is decreased, as was described by Mandelbrot, whereas the FD always remains the same. The FD can only be used as a surrogate for the boundary length over the assumption that the magnification of the applied method is capable of detecting the smallest possible particles of the object. The used real-time MRI technique represents the state of scientific and technological progress and the determined FD values are therefore equated with the boundary length. However, different results may be obtained should real-time MRI with higher spatial resolution become available. The information of FD evolution of a disintegrating tablet allows for a circumstantial investigation of the disintegration process. Due to the high temporal resolution of the conducted real-time MRI experiments, the disintegration behavior of rapidly disintegrating tablets can also be evaluated.

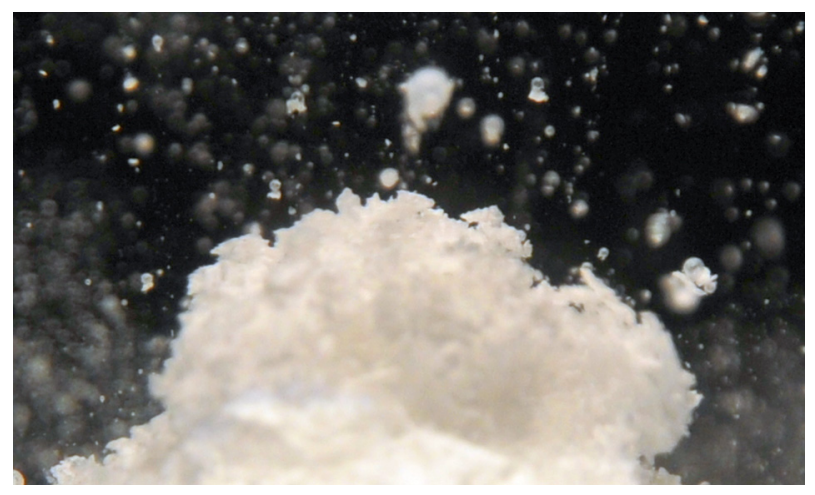

Fig. 2. Digital image of disintegrating XPVP tablet. 
Real-time MRI techniques yield dynamic series of cross-sectional images (i.e., intensity distributions) that represent arbitrarily oriented thin sections through a sample. Dynamic analyses of sections have the advantage to avoid signal contributions from tablet particles that originate from different areas of the tablet. In fact, if a tablet is photographed from the outside during disintegration, one cannot distinguish from which part of the tablet the particles contribute to the recorded surface geometry (Fig. 2). This can lead to unrealistic large FDs, in case of rough particles contributing to the tablet outline, or to too small FDs, when overlapping particles create a seemingly smooth surface.

In a previous study, the acquired MR images were evaluated by a qualitative and quantitative analysis of the respective histograms (Quodbach et al., 2014). The images allowed for a differentiation between the mechanisms of action of the disintegrants, whereas the histograms yield information about the distribution and relative amount of water within a tablet during disintegration.

In this work a novel algorithm for data analysis is presented to calculate the FDs of tablet boundaries. The fractal dimension is introduced as a surrogate parameter for the available surface area of a tablet and thus emerges as a novel measure for the effectiveness of disintegrants, which can be calculated directly from the MRI data. The impact and influence of different disintegrants, of varying relative densities and of disintegrant concentrations are investigated and the method's performance discussed.

\section{Materials and methods}

\subsection{Materials}

The investigated disintegrants were: two sodium starch glycolates (Explotab, JRS Pharma, Rosenberg, Germany, and Primojel, DMV-Fronterra Excipients, Goch, Germany), two polacrilin potassium resins (Amberlite IRP88, Rohm and Haas France SAS, Chauny, France, and Kyron T-314, Corel Pharma Chem, Ahmedabad, India), crospovidone (Polyplasdone XL, ISP, Waalwijk, Netherlands), and croscarmellose sodium (Ac-Di-Sol FMC BioPolymer, Wallingstown, Ireland). Dibasic calcium phosphate (Di-Cafos C92-14, Chemische Fabrik Budenheim, Budenheim, Germany) was chosen as filler due to its hydrophilic properties but insolubility in water. Magnesium stearate (Parteck LUB MST, Merck, Darmstadt, Germany) was added as lubricant. Abbreviations are shown in Table 1 and will be used to specify the different tablet batches (e.g., tablets containing SSG I will simply be referred to as SSG I).

\subsection{Blend and tablet preparation, disintegration time measurements}

Blends were prepared with a Turbula-Blender (T2C, W.A. Bachofen AG, Basel, Switzerland). After $10 \mathrm{~min}$ of blending, 0.5\% of magnesium stearate was added to each mixture before mixing was continued for $2 \mathrm{~min}$. Blends for three experimental parts were prepared. The first blend consisted of $97.5 \%$ dibasic calcium phosphate and $2 \%$ of CCS. The second series of blends contained $0.5 \%$ to $16 \%$ of PP I and dibasic calcium phosphate. The

Table 1

Abbreviations of used excipients.

\begin{tabular}{lll}
\hline Brand name & Substance & Abbreviation \\
\hline Explotab & Sodium starch glycolate & SSG I \\
Primojel & Sodium starch glycolate & SSG II \\
Amberlite IRP88 & Polacrilin potassium & PP I \\
Kyron T-314 & Polacrilin potassium & PP II \\
Polyplasdone XL & Crospovidone & XPVP \\
Ac-Di-Sol & Croscarmellose sodium & CCS \\
Di-Cafos C92-14 & Dibasic calcium phosphate & DCP \\
\hline
\end{tabular}

blends for the last part were prepared with $97.5 \%$ of dibasic calcium phosphate and $2 \%$ of different disintegrants. The disintegrant concentration of $2 \%$ was chosen as it is within the concentration ranges of the applied disintegrants as proposed by the manufacturers. Common disintegrant concentrations range between $0.5 \%$ and $5 \%$, but can be higher when certain requirements have to be met, e.g., for orally disintegrating tablets.

Tablets were compressed on a rotary die press (Pressima MX Eu-B/D, IMA Kilian, Cologne, Germany) with $10 \mathrm{~mm}$ flat faced punches. The die was filled manually with $700 \mathrm{mg}$ of powder blend. The tablet height was adjusted to compress tablets of a relative density $(\rho)$ of 0.85 (experimental parts two and three), or of relative densities ranging from 0.65 to 0.85 in 0.05 steps (first experimental part). Tablets were stored at $45 \%$ relative humidity and $21^{\circ} \mathrm{C}$.

The disintegration times were measured either 3 months after production (comparison of disintegrants) or 2 weeks after production (varying relative density of CCS and different concentrations of PP I) with a SOTAX DT2 (SOTAX GmbH, Loerrach, Germany) according to the European Pharmacopoeia (European Pharmacopoeia 8.1, 2014). The storage time strongly influences the disintegration time as shown in Table 2 . Therefore, the data is only consistent within a given measurement set (varying relative density of CCS, increasing amounts of PP I, and different disintegrants) and the disintegration times cannot be compared between data sets.

\subsection{Real-time MRI}

Measurements were performed on a 9.4 T MRI system (Bruker Biospin, Ettlingen, Germany) with a Bruker BGA12S gradient system (inner bore $118 \mathrm{~mm}$ ) yielding a maximum gradient strength of $660 \mathrm{mT} \mathrm{m}^{-1}$ and slew rate of $4570 \mathrm{~T} \mathrm{~m}^{-1} \mathrm{~s}^{-1}$. Mildly T1-weighted real-time MRI at 13.3 frames per second was accomplished with use of an undersampled radial FLASH sequence and regularized nonlinear inversion (NLINV) for image reconstruction (Uecker et al., 2010; Zhang et al., 2010). To reduce image artifacts that may be caused by an inaccurate technical switching of the radial encoding gradients, linear and $B_{0}$ eddy-current effects were corrected by methods described by Block and Uecker (2011) and Moussavi et al. (2014).

\section{Table 2}

Maximum FD values with according time points, as well as disintegration times of investigated formulations ( $n=6$; mean $\pm \mathrm{CI}, \alpha=0.05$ ).

\begin{tabular}{lccc}
\hline & Time of maximum FD/s & Maximum FD & Disintegration time/s \\
\hline CCS & & & \\
$\rho=0.65$ & 3.6 & $1.45 \pm 0.03$ & $17.5 \pm 1.4$ \\
$\rho=0.70$ & 3.1 & $1.48 \pm 0.01$ & $16.2 \pm 1.2$ \\
$\rho=0.75$ & 3.0 & $1.46 \pm 0.01$ & $7.9 \pm 0.4$ \\
$\rho=0.80$ & 4.1 & $1.48 \pm 0.02$ & $5.7 \pm 0.9$ \\
$\rho=0.85$ & 6.6 & $1.45 \pm 0.02$ & $6.6 \pm 0.8$ \\
& & & \\
PP I & & & \\
$0 \%$ & 2.8 & $1.24 \pm 0.06$ & $>180.0$ \\
$0.5 \%$ & 11.0 & $1.40 \pm 0.07$ & $88.3 \pm 50.1$ \\
$1 \%$ & 5.7 & $1.41 \pm 0.02$ & $13.1 \pm 7.1$ \\
$2 \%$ & 2.6 & $1.45 \pm 0.01$ & $5.3 \pm 0.6$ \\
$4 \%$ & 3.5 & $1.50 \pm 0.03$ & $6.3 \pm 0.8$ \\
$8 \%$ & 2.9 & $1.56 \pm 0.02$ & $7.0 \pm 0.8$ \\
$16 \%$ & 2.4 & $1.54 \pm 0.04$ & $10.9 \pm 1.1$ \\
& & & \\
Disintegrant & & & \\
SSG I & 24.9 & $1.43 \pm 0.02$ & $46.5 \pm 1.7$ \\
SSG II & 22.8 & $1.48 \pm 0.02$ & $47.8 \pm 4.2$ \\
PP I & 2.6 & $1.45 \pm 0.01$ & $19.6 \pm 4.6$ \\
PP II & 11.6 & $1.42 \pm 0.02$ & $14.9 \pm 0.9$ \\
XPVP & 4.1 & $1.45 \pm 0.01$ & $17.1 \pm 2.2$ \\
CCS & 6.6 & $1.45 \pm 0.02$ & $15.3 \pm 0.4$ \\
\hline
\end{tabular}


Dynamic acquisitions were performed with a repetition time $\mathrm{TR}=3.0 \mathrm{~ms}$, echo time $\mathrm{TE}=1.8 \mathrm{~ms}$, and flip angle $=5^{\circ}$. The measured field of view of $25.6 \mathrm{~mm} \times 25.6 \mathrm{~mm}$ was covered with an in-plane resolution of $80 \mu \mathrm{m} \times 80 \mu \mathrm{m}$ and a slice thickness of $600 \mu \mathrm{m}$. Accordingly, the acquired images have an in-plane resolution of $320 \times 320$ pixels. Temporal resolution was $75 \mathrm{~ms}$ using 25 radial spokes per frame.

\subsection{MR-compatible measurement cavity}

Identical manipulation of tablets was assured by a custom-made device tailored to the Bruker gradient system. A phased-array surface coil below the cavity ensured a maximum signal, i.e., a minimum coil-to-tablet distance. The tabletlaunching device was loaded by placing a single tablet within a chute in the cap of the cavity above the disintegration medium. The cavity itself contained $10 \mathrm{ml}$ of demineralized water (doped with $0.1 \mathrm{mg} / 100 \mathrm{ml} \mathrm{CuSO}_{4}$ solution $4 \mathrm{mM}$ ). A semiautomatic tablet release mechanism manually controlled from outside the magnet room allowed for imaging of the tablet disintegration process within the magnet bore. After the tablets were released, they dropped into the water and the disintegration started.

\subsection{Data analysis}

MATLAB R2012b (MathWorks, Natick, USA) was used to analyze the reconstructed images. A box-counting algorithm estimated the FDs of the binary images. The box-counting algorithm applied in this study performed a fixed-grid scan with box sizes of $2^{\mathrm{p}}$ pixels using $p=1-7$. During a fixed-grid scan, boxes of varying sizes are placed side-by-side on object boundaries in the image. The number of boxes covering a boundary increases with decreasing box size. If a morphological structure has fractal properties, a straight line results in a log-log plot of the box sizes versus the number of boxes (Fig. 3 ).

The negative slope of the linear regression of this line represents an estimation of the FD of the investigated image referred to as FD in the following. The box size of $1 \times 1$ pixels is excluded in the linear regression because it represents only the number of boundary pixels in the images and does not add information about the surface geometry. An introduction into the topic of fractal geometry and analysis with descriptions of different estimation algorithms is given by Kaye (Kaye, 1994). Images were cropped prior to box-count analysis to $186 \times 111$ pixels. If the box size of the box-count analysis exceeds the image dimensions, the algorithm assumes the missing pixels as 0 , thus not altering the estimated result. At the moment when the tablets drop into water, the FDs were estimated to be

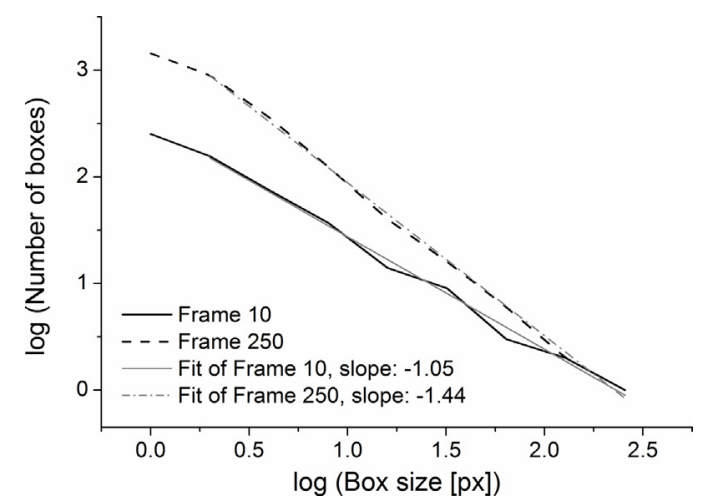

Fig. 3. Box-counting data (log-log plot) for two different frames of a tablet containing explotab (grey lines = linear regression of plots). unexpectedly high before decreasing to lower values. The detected signal is caused by the inflow phenomenon. $1 \mathrm{H}$ nuclei that are not in the imaging plane are not exposed to the repetitive slice-selective radio frequency excitations from the emitter coil. When they flow into the imaging plane after the tablet dropped into the water, they are freshly excited and therefore create a strong signal due to their unsaturated magnetization. This phenomenon lasts less than $0.5 \mathrm{~s}$ and is also visible in the Supplementary videos. Analysis of images showing the inflow phenomenon causes the detection of falsely high FDs, as the artifacts are detected by the box-counting algorithm. Subsequently, the FDs drop to a minimum when the artifacts disappear and rise afterwards due to the disintegrant action. The time point of the minimum was defined as time point 0 in all cases. Data prior to the minimum is not shown.

See Excel sheet 1 as supplementary file. Supplementry material related to this article found, in the online version, at http://dx.doi. org/10.1016/j.ijpharm.2014.09.021.

Air bubbles are released from the tablet matrices in some measurements, which are analyzed as part of the tablet surface structure (e.g., see Supplementary Video 2 of a SSG II containing tablet). This could alter the FD, yet, as it was not investigated what the FD of the surface of an air bubble imaged with MRI is, it cannot be said whether the average FD would increase of decrease. Working under a low pressure atmosphere could prevent the entrapment of air within the tablet matrix. However, no low pressure system was established for the presented measurements. Therefore, the results are interpreted as they are under the premise that all measurements are equally affected.

To determine the maximum FD values, only the first $15 \mathrm{~s}$ of data were analyzed to avoid plateau regions with constant values. For SSG I and II, the complete measurement data was used as they disintegrate more slowly compared to the other disintegrants. Analysis was performed with the software OriginPro $8.5 \mathrm{G}$ (OriginLab Corporation, Northampton, USA).

\section{Results and discussion}

\subsection{Data treatment}

Prior to data treatment, an area of interest for the FD determination had to be defined. In order to measure the influence of the disintegrant on the evolution of disintegration, a continuously changing surface is necessary. The only region fulfilling this requirement is the upper boundary of the tablet, as detached particles settle on the sides around the tablet band and superimpose the changes there (Fig. 4A). Therefore, the images were cropped to the same size at the upper tablet edge for further analysis (Fig. 4B). By adding an inverted image from the beginning of each measurement without a tablet to every following image, the decreasing coil sensitivity is suppressed (Fig. 4C). Subsequently, a threshold (gray value) is calculated for every image with Otsu's method (Otsu, 1979). This algorithm calculates the threshold by searching for the lowest intra-class variance between foreground and background pixels. The calculated threshold is used for the subsequent binarization. The binary image was inverted, an erosion/dilation performed, and multiplied with the original binary image. The size of the rectangular structural element for the erosion was set to $3 \times 3$ pixels, the size of the dilation element was set to $6 \times 6$ pixels. After the multiplication step of the image matrices, the surface information remains unchanged, while artifacts smaller than $3 \times 3$ pixels are removed (Fig. 4D). The last step was the application of the Sobel edge detection algorithm, which provided the overall best match with the binarized image (Fig. 4E). 

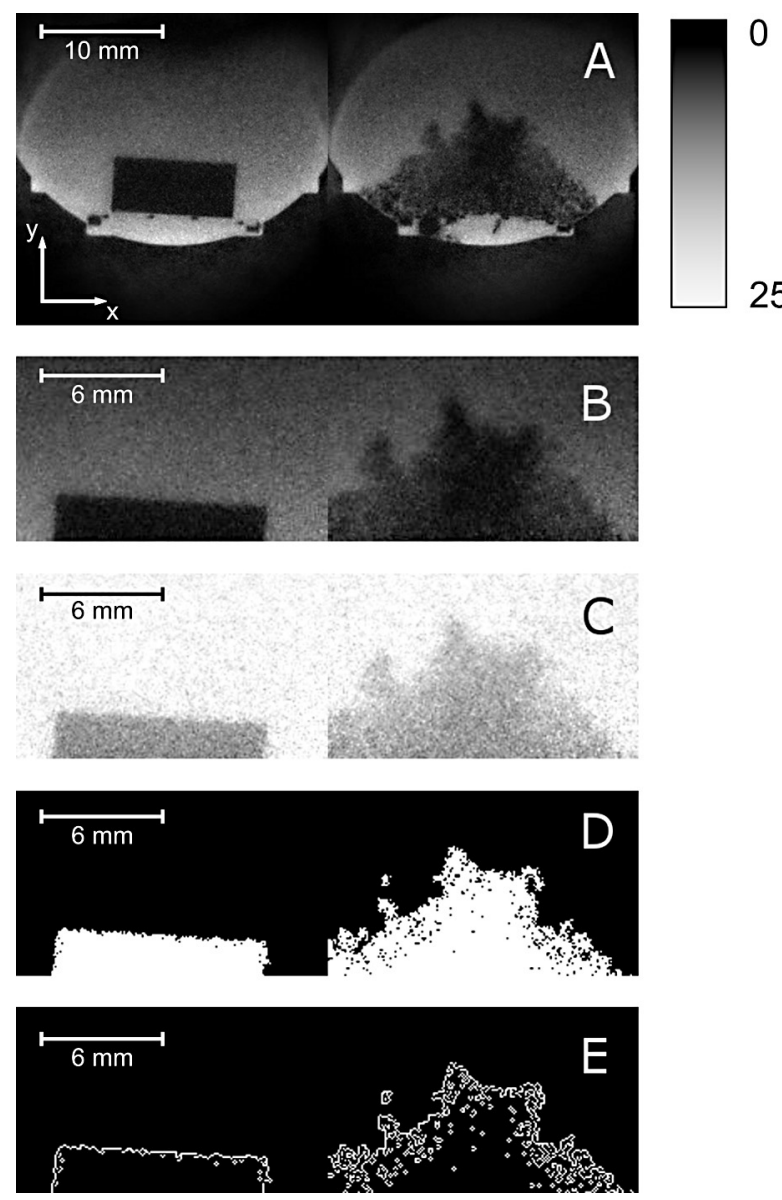

Fig. 4. Steps of data treatment at two different time points of disintegration; A: MRI input, B: region of interest, C: coil sensitivity correction, D: binarization, E: edge detection; the intensity bar applies only to images A-C.

The most crucial step within this data treatment is the binarization following the intensity correction of the data. Fig. 5 displays a comparison of input and output data from Otsu's algorithm and subsequent binarization. The algorithm works best on strong edges as displayed in Fig. 5A1. The penetrating water in Fig. 5A2 and A3 causes lighter gray values at the edges of the tablet, resulting in a more complicated surface geometry. The binarization with the calculated threshold value represents the set structure of the not disintegrated tablet without apparent changes (Fig. 5B1). The boundaries of the further disintegrated tablets, however, show a greater loss in detail. Pixels of a light gray are excluded during binarization (Fig. 5B2 and B3), leading to a more rugged surface representation than suggested by the original data. Although the FD estimation depends on the spatial resolution (Huang et al., 1994), no influence of this effect is expected because all images were of the same size. Different fractal dimensions would only be expected when the image size and therefore the resolution of the portrayed particles changed during the measurement. All data was treated with the described edge-extraction and box-counting algorithm.

It has to be considered that multiple particles can overlap in the imaging plane, as the slice thickness is $600 \mu \mathrm{m}$. This influences the data in two ways. Firstly, the more particles overlap, the lower the intensity will be in the specific region (visible as darker pixels). The intensity is lowest when the volume of the measurement plane is devoid of water and filled with tablet particles, causing an intensity value similar to that of the not-disintegrated tablet. When only a small, single particle is present in the measurement slice, the signal from the water also present in the acquisition volume will increase the overall signal and cause a soft transition from the particle to the water signal. Secondly, it cannot be pointed out whether one or several particles are projected on the imaging plane. Therefore, the in-plane resolution of $80 \mu \mathrm{m} \times 80 \mu \mathrm{m}$ can only be interpreted together with the slice thickness defining an acquisition volume. However, depending on the number of overlapping particles, the surface structure can ideally be resolved with a resolution of $80 \mu \mathrm{m} \times 80 \mu \mathrm{m}$. Yet it has to be kept in mind that the measurement is not two dimensional but covers a volume. Thus, the resolution cannot be exactly specified even though the in-plane resolution is clearly defined.

\subsection{Fractal dimensions of tablets with varying relative densities containing CCS}

The investigated tablets represent the range of relative densities of typical pharmaceutical tablets (Hancock et al., 2003). The following discussions are based on the mean curves as they represent the kinetics and values of the curves of the single measurements well. The FDs of tablets with a relative density ranging from $\rho=0.65$ to $\rho=0.8$ reach their maximum of approximately 1.46 after around $3-4 \mathrm{~s}$ (Fig. 6). Tablets of $\rho=0.85$ require $6.6 \mathrm{~s}$, almost twice as long, to peak at a FD of 1.45 (Table 2). The maxima are followed by a decline and larger confidence intervals because of some particles dropping out of the measurement plane sometimes, leaving a slightly smoother surface behind. The decline is more pronounced for tablets of
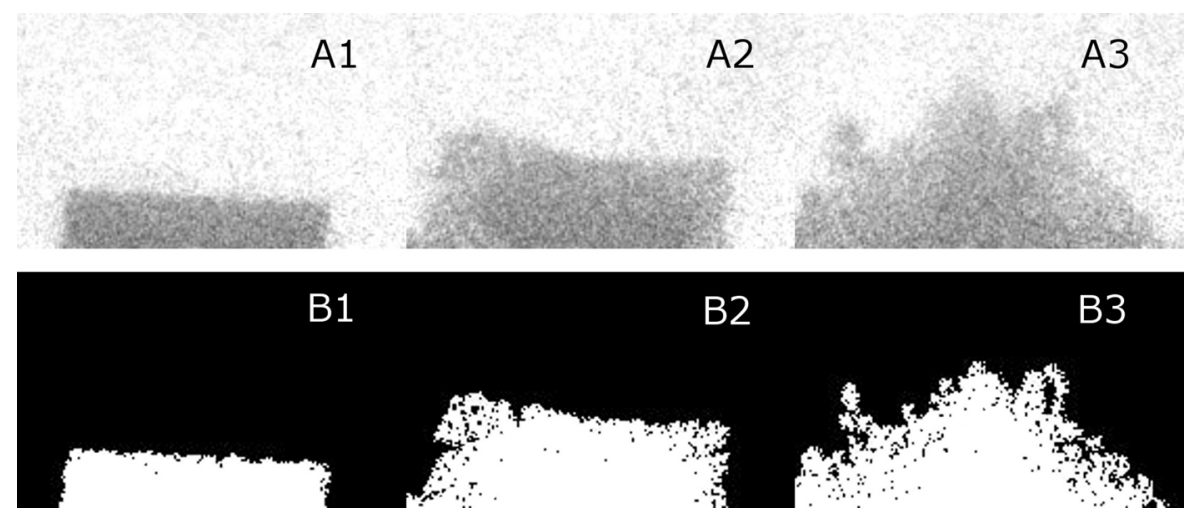

Fig. 5. Before (A1-A3) and after (B1-B3) binarization after thresholding with Otsu's method. 


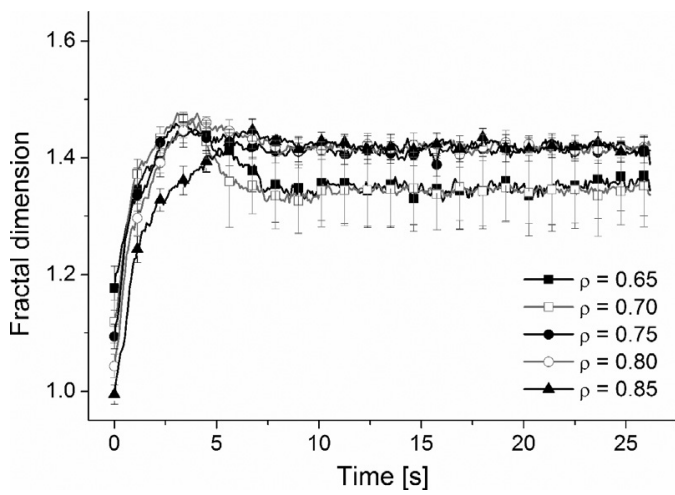

Fig. 6. Fractal dimensions for different relative density of CCS ( $n=5$ or $6 \pm \mathrm{CI}$, $\alpha=0.05)$.

the two lowest relative densities, as larger chunks of the upper part drop out of the region of interest selected for analysis. This results in a drop of FDs as well as increasing confidence intervals. If the mean of the FDs of the two lowest relative densities is calculated solely with the cases when all particles remain in the plane of view, the values are similar to those of higher relative densities (not shown). A possible explanation for the slower kinetics of tablets with $\rho=0.85$ might be given by the Washburn equation for horizontal capillaries (Washburn, 1921). According to the equation, the penetration rate is directly proportional to the pore radius. The large span between lowest and highest relative densities resulted in tabletting pressures roughly between $60 \mathrm{MPa}$ and $380 \mathrm{MPa}$. The average pore size will be greatly decreased at high pressures, leading to slower penetration rates at high relative density. Yet it cannot be explained why this does not affect tablets of lower relative density. Another possible reason could be that the porous system does not percolate the complete tablet at the highest relative density, which would also delay the water penetration. Yet it cannot be concluded what the actual reason for the observed behavior is. The results suggest a negligible impact of varying relative density between $\rho=0.65$ and $\rho=0.8$ on the efficacy of CCS and only a minor kinetic change at $\rho=0.85$.

Based on the results of List and Muazzam, who reported a decreasing disintegration time with increasing relative density (List and Muazzam, 1979), which is in agreement with own results (Table 2), larger FDs were expected in combination with higher relative density. The authors explained this behavior with the decreasing void space in tablets compressed to higher relative density. This enables the disintegrant particles to exert more force onto the tablet matrix. Yet the FD results show that disintegration propensity does not correlate with the detected boundary roughness. In previous work, however, the degree of distribution and amount of water in the tablet matrix depended on the relative density - higher relative densities leading to a more even distribution of a higher amount of water within the tablet (Quodbach et al., 2014). Therefore, surface area and amount of water do not necessarily depend on each other.

\subsection{Fractal dimensions of tablets containing different amounts of PP I}

Contrary to the results of varying relative density, height and rate at which the maximum FD is reached depend greatly on the concentration of PP I (Fig. 7). At a concentration of $0.5 \%$, $11 \mathrm{~s}$ are necessary to peak at a FD of 1.4 , whereas $1 \%$ of disintegrant reaches the maximum in $5.7 \mathrm{~s}$ (Table 2). Concentrations of $2 \%$ and higher reach their highest FD after $\sim 3 \mathrm{~s}$, indicating a kinetic limit for the initial disintegration process. This could be due to a limited water penetration rate into the porous tablet. The hydrophilic properties

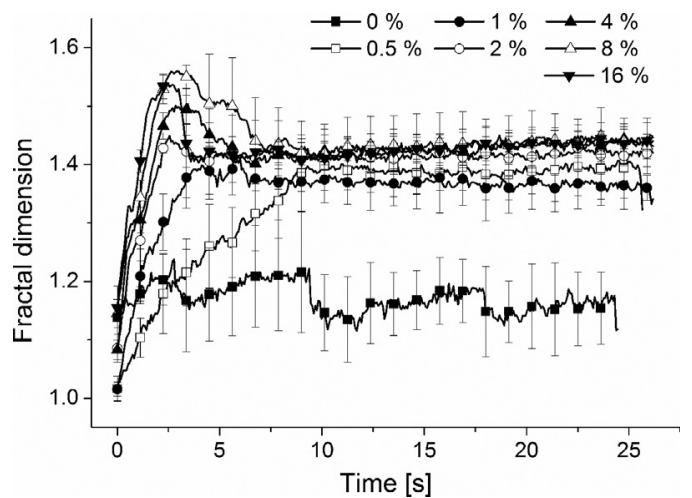

Fig. 7. Fractal dimensions for different concentrations of PP I ( $n=5$ or $6 \pm \mathrm{CI}$, $\alpha=0.05,0 \%: n=3 \pm \mathrm{SD}$ ).

of the disintegrant polymer are likely able to increase the water penetration rate until the viscosity of the water limits further improvements. The maximum FD, however, increases with larger amounts of disintegrants. The maximal FD of tablets with $2 \%$ is 1.45 , with $4 \% 1.5$, with $8 \% 1.56$, and with $16 \% 1.54$. Above $0.5 \%$, the FDs drop visibly after following its maximum. This is, as described before, due to particles falling out of the covered plane. The lower FD of $16 \%$ tablets compared to $8 \%$ tablets is likely caused by the same phenomenon. However, the concentration of PP I has a major impact on the performance of a disintegrant. The effects of the single disintegrant particles accumulate, leading to a larger accessible surface area at high concentrations.

While a relationship between disintegration time and timeto-maximum FD is evident, the absolute differences are larger in the case of disintegration time $(5.3 \pm 0.6-88.3 \pm 50.1 \mathrm{~s}$ compared to $2.4-11.0 \mathrm{~s}$ ). The disintegration time is lowest for a disintegrant concentration of $2 \%$ and is highest for a concentration of $0.5 \%$. At even higher concentrations, the disintegration time increases slightly, pointing towards the good binding properties of PP I. The time-to-maximum FD decreases from $11.0 \mathrm{~s}$ to $2.4 \mathrm{~s}$ with the exception of the $2 \%$ batch, which reacts equally fast as the $16 \%$ samples. The short time in which tablets without disintegrant reach the maximum $\mathrm{FD}$ is due to air bubbles attached to the surface of the tablet. The tablets do not disintegrate and are therefore not discussed.

Low disintegrant concentrations lead to a slower disintegration and a longer time span to reach the maximum FD. Therefore, the available surface area is expected to be lower when less disintegrant is applied.

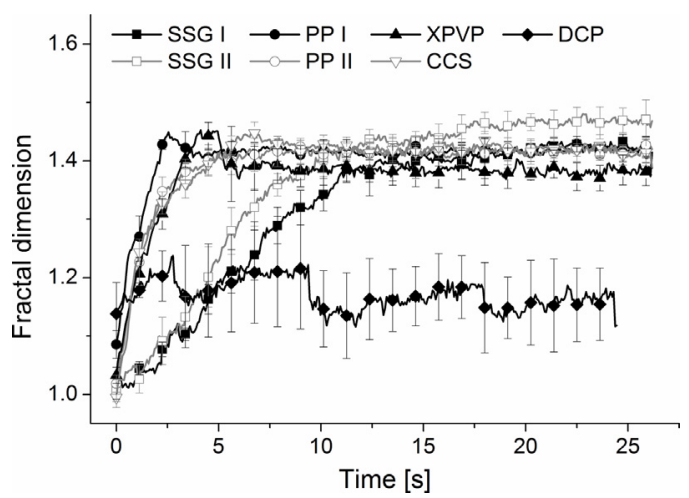

Fig. 8. Fractal dimensions of different disintegrants $(n=5$ or $6 \pm \mathrm{CI}, \alpha=0.05$, DCP: $n=3 \pm$ SD) 
Table 3

Rank order of disintegrants according to the evolution velocity of FDs (fastest to slowest).

\begin{tabular}{ll}
\hline Rank & Disintegrants \\
\hline 1 & PP I, XPVP \\
2 & PP II, CCS \\
3 & SSG I, SSG II \\
\hline
\end{tabular}

\subsection{Fractal dimensions of tablets containing different disintegrants}

Even though the FDs are of similar magnitude, three groups of disintegrants can be differentiated by their kinetics (Fig. 8). The most distinct group comprises SSG I and II. Their FDs rise slowest and have not reached a steady state before $22 \mathrm{~s}$ (Table 2 ). It has been reported that SSG may cause an increase of the fluid viscosity (Shah and Augsburger, 2002) due to non-linked monomers remaining from the polymer synthesis. This can cause the formation of a viscous barrier within the tablet core, slowing down the water penetration and, thus, the disintegration process. SSG II, however, reaches the highest FD of 1.48. Surprisingly, the second and third group both contain disintegrants of different chemical structures. It was expected that the kinetics of different brands of the same chemical substances resemble one another, so that PP I and PP II should display similar characteristics. However, the kinetics PP I resemble that of XPVP and PP II resembles CCS. PP I and XPVP display a steep increase of FD until the maxima are reached after $2.5 \mathrm{~s}$ (PP I) or $4 \mathrm{~s}$ (XPVP), respectively. The curve shape of PP II and CCS is similar to that of PP I and XPVP within the first $2 \mathrm{~s}$ but begins to flatten afterwards, having their largest FDs at $7 \mathrm{~s}$ (CCS) and $8 \mathrm{~s}$ (PP II). The MRI videos of the disintegrating tablets illustrate the results (online supplement). PP I and XPVP react quicker and show a stronger vertical expansion than PP II and CCS. A reason for this might be differing mechanisms of action of PP I and PP II, which has to be investigated further. Possible explanations might be different degrees of crosslinking or different crosslinking modes (i.e., chemical or physical crosslinking). Pure DCP seems not to be as readily wetted as a mixture with disintegrant, causing several air bubbles to be attached to the surface, which leads to unexpectedly high FDs. Also, some air is released out of the tablets within the first few seconds and remains attached to the tablet surface, increasing the FDs further to values around 1.2. The subsequent drops of FDs are due to bursting or detaching air bubbles.

The data present only minor differences in the absolute value of the FDs but large variations in the kinetics. This suggests an independency of the FD as surrogate for the accessible surface area from the choice of disintegrant at a concentration level of $2 \%$. A rank order of disintegrant efficiency concerning the disintegration kinetics is shown in Table 3.

The times in which the tablets reach the maximum FDs mostly comply with the measured disintegration times (Table 2). SSG I and II require three to four times longer to disintegrate completely and develop their maximum FD slower than the other disintegrants. The absolute values of disintegration times are larger in general, since the whole tablet needs to disintegrate instead of a change of only the surface.

Di basic calcium phosphate based tablets exhibit a very rapid disintegration where differences in the kinetics might not be properly differentiated. If another matrix substance was used, the impact on the disintegration process could be more pronounced.

\section{Conclusion}

This study proposes a novel method to quantify the efficacy of disintegrants by means of fractal dimensions. Prior studies were not able to provide comparable data on the role disintegrants play in generating large surface areas in little time. Real-time MRI enables the acquisition of data that hitherto was either not accessible or required several different experiments. In this study, a coherent set of efficacy parameters for 6 disintegrants of 4 different chemical structures was evaluated by real-time MRI at high spatial resolution. Because the formulation was chosen to influence the disintegration process as little as possible, the data represents mainly the properties of the investigated disintegrants. The new method was not able to sufficiently differentiate between tablets of varying relative density, whereas the detected impact of changing disintegrant concentrations and varying disintegrants demonstrate the potential of the proposed method to acquire a wide range of data in a single experiment.

It has to be considered that the graphs of the FDs lose some of their distinctive character after reaching the maximum. The cumulative effect of the remaining disintegrant particles cannot compensate for particles dropped out of the imaging plane, which causes apparently lower FD estimates. In consequence, the graphs can only be analyzed up to the maximum and therefore only cover the initial phase rather than the complete course of the disintegration process. Further analysis of this phenomenon will only be possible when multiple sections of a given tablet can be recorded at the same time with similar spatiotemporal resolution. Furthermore, the experimental setup is static and does not represent the stress exerted on a tablet in the stomach. It needs to be investigated how different matrix substances, e.g. water-soluble lactose, influence the disintegration efficacy and if other disintegrants are also insensitive to changes in relative density.

\section{Conflict of interest}

The authors declare that there is no conflict of interests.

\section{Acknowledgements}

Part of this work (R.T.) was supported by the Cluster of Excellence and DFG Research Center for Nanoscale Microscopy and Molecular Physiology of the Brain.

\section{References}

Block, K., Uecker, M., 2011. Simple method for adaptive gradient-delay compensation in radial MRI. 19th Annual Meeting ISMRM, Montreal, Quebec, Canada.

Caramella, C., 1990. Novel methods for disintegrant characterization, part II. Pharm. Technol. Int. 30-37.

Chen, C.R., Lin, Y.H., Cho, S.L., Yen, S.Y., Wu, H.L.S., 1997. Investigation of the dissolution difference between acidic and neutral media of acetaminophen tablets containing a super disintegrant and a soluble excipient. Chem. Pharm. Bull. 45, 509-512.

European Pharmacopoeia 8.1, 2014. In: Commission, E.P. (Ed.), Disintegration of Tablets and Capsules. European Directorate for the Quality of Medicines (EDQM), Strasbourg, France, pp. 285-287.

Gissinger, D., Stamm, A., 1980. A comparative evaluation of the properties of some tablets disintegrants. Drug Dev. Ind. Pharm. 6, 511-536.

Gordon, M.S., Chowhan, Z.T., 1990. The effect of aging on disintegrant efficiency in direct compression tablets with varied solubility and hygroscopicity, in terms of dissolution. Drug Dev. Ind. Pharm. 16, 437-447.

Hancock, B.C., Colvin, J.T., Mullarney, M.P., Zinchuck, A.V., 2003. The relative densities of pharmaceutical powders, blends, dry granulations, and immediaterelease tablets. Pharm. Technol. 27, 64-80.

Huang, Q., Lorch, J.R., Dubes, R.C., 1994. Can the fractal dimension of images be measured? Pattern Recogn. 27, 339-349.

Iwao, Y., Tanaka, S., Uchimoto, T., Noguchi, S., Itai, S., 2013. An easy-to-use approach for determining the disintegration ability of disintegrants by analysis of available surface area. Int. J. Pharm. 448, 1-8.

Jyothi, G., Lakshmi, P.K., 2011. Comparative evaluation of natural and synthetic superdisintegrants with newer superdisintegrant Kyron T-314. Acta Pharm. Sci. $53,35-44$.

Kaye, B.H., 1994. A Random Walk Trough Fractal Dimensions, second ed. VCH Verlagsgesellschaft, Weinheim, Germany. 
List, P.H., Muazzam, U.A., 1979. Quellung - die treibende kraft beim tablettenzerfall, 2. Mitteilung Pharm. Ind. 41, 1075-1077.

Mandelbrot, B., 1967. How long is the coast of Britain? Statistical self-similarity and fractional dimension. Science 156, 636-638.

Mesnier, X., Althaus, T.O., Forny, L., Niederreiter, G., Palzer, S., Hounslow, M.J., Salman, A.D., 2013. A novel method to quantify tablet disintegration. Powder Technol. 238, 27-34.

Moussavi, A., Untenberger, M., Uecker, M., Frahm, J., 2014. Correction of gradientinduced phase errors in radial MRI. Magn. Reson. Med. 71, 308-312.

Otsu, N., 1979. Threshold selection methods from gray-level histograms. IEEE Trans. Syst. Man Cyb. SMC 9, 62-66.

Quodbach, J., Moussavi, A., Tammer, R., Frahm, J., Kleinebudde, P., 2014. Tablet disintegration studied by high-resolution real-time magnetic resonance imaging. J. Pharm. Sci. 103, 249-255.

Rudnic, E.M., Rhodes, C.T., Welch, S., Bernardo, P., 1982. Evaluations of the mechanism of disintegrant action. Drug Dev. Ind. Pharm. 8, 87-109.
Shah, U., Augsburger, L., 2002. Multiple sources of sodium starch glycolate, NF: evaluation of functional equivalence and development of standard performance tests. Pharm. Dev. Technol. 7, 345-359.

Shotton, E., Leonard, G.S., 1972. Effect of intra- and extra-granular maize starch on disintegration of compressed tablets. J. Pharm. Pharmacol. 24, 798-803.

Soumya, M.S., 2013. Formulation and invitroevaluation of fast dissolving tablets of flecainide acetate. Int. J. Pharm. Pharm. Sci. 5, 555-560.

Uecker, M., Zhang, S., Voit, D., Karaus, A., Merboldt, K.-D., Frahm, J., 2010. Real-time MRI at a resolution of $20 \mathrm{~ms}$. NMR Biomed. 23, 986-994.

Washburn, E.W., 1921. The dynamics of capillary flow. Phys. Rev. 17, 273.

Zhang, S., Block, K., Frahm, J., 2010. Magnetic resonance imaging in real time: advances using radial FLASH. J. Magn. Reson. Imag. 31, 101-109.

Zhao, N., Augsburger, L., 2005. Functionality comparison of 3 classes of superdisintegrants in promoting aspirin tablet disintegration and dissolution. AAPS PharmSciTech 6. 